\title{
Digital Implementation of Fuzzy Logic Controller for Real Time Position Control Applications
}

\author{
Umarani $\mathrm{P}^{\# 1}$, Vasanthmohan $\mathrm{S}^{\# 2}$ \\ ${ }^{1}$ Department of Electronics and Communication Engineering, Mount Zion College of Engineering \& \\ Technology/Anna university, India' \\ ${ }^{2()}$ Department of Electronics and Communication Engineering, Mount Zion College of Engineering \& \\ Technology/Anna university, India)
}

\begin{abstract}
Fuzzy Logic Controller (FLC) systems have emerged as one of the most promising areas for Industrial Applications. The highly growth of fuzzy logic applications led to the need of finding efficient way to hardware implementation. Field Programmable Gate Array (FPGA) is the most important tool for hardware implementation due to low consumption of energy, high speed of operation and large capacity of data storage. In this paper, instead of an introduction to fuzzy logic control methodology, we have demonstrated the implementation of a FLC through the use of the Very high speed integrated circuits Hardware Description Language (VHDL) code. FLC is designed for position control of BLDC Motor. VHDL has been used to develop FLC on FPGA. A Mamdani type FLC structure has been used to obtain the controller output. The controller algorithm developed synthesized, simulated and implemented on FPGA Spartan 3E board.
\end{abstract}

Keywords - BLDC Motor, FLC, Hardware Implementation, Spartan 3 FPGA, VHDL

\section{Introduction}

The past few years have witnessed a rapid growth in the number and variety of application of fuzzy logic. The application ranges from consumer products such as cameras, washing machines, cars and in industry for medical instrumentation, underground trains and robots. Unlike the conventional controller FLC design is not based on the mathematical model of the plant or system. A FLC is an automatic controller that controls an object in accordance with desire behaviour. For a complex system whose mathematical model is very difficult to define or the transfer function of a plant is undefined, fuzzy logic controllers are very useful in that case $[1,2]$. The control action of FLC is defined in terms of simple human friendly "if - then rules". These set of rules are describe the system behaviour. These set of rules are called the knowledge base of fuzzy controller. We can easily change the rules accordance with our desire output. So the development time for a new controller can be significantly reduced as compared to conventional one [3]. The motivation behind the implementation of a FLC in VHDL was driven by the need for an inexpensive hardware implementation of a generic fuzzy controller for use in industrial and commercial applications [4]. We have taken a simple FLC for position control of BLDC motor. Position Error and Angular position has been used as two inputs to FLC. For both the inputs 3 Gaussian membership function has been selected and coded in VHDL. An algorithm has been developed in VHDL to fuzzify the crisp digital values of Position error and Angular position. Mamdani type FLC structure has been used to obtain the controlled output. The controller algorithm developed synthesized, simulated and implemented on FPGA Spartan 3E board. The FLC has been design using system generator approach. The results of the FLC implemented on FPGA have been compared with the results obtained using FLC on MATLAB Simulink.

\section{System Description}

A Brushless DC motor has a permanent magnet rotor and a wound stator. Furthermore, there are two types of brushless motors; the type that has an outer rotating magnet or the type that has an inner rotating magnet assembly. In a brushless DC motor the position of the coils (phases), with respect to the permanent magnet field, are sensed and the current switched electronically (commutated) to the appropriate phases. Hall Effect sensors are typically used to sense the rotor position. The Brushless Direct Current (BLDC) motor is rapidly gaining popularity by its utilization in various industries, such as Appliances, Automotive, Aerospace, Consumer, Medical, Industrial Automation Equipment and Instrumentation. As the name implies, the BLDC motors do not use brushes for commutation; instead, they are electronically commutated [5] [6]. The BLDC motors have many advantages over brushed DC motors and induction motors. A few of these are Better speed versus torque response, High dynamic response, High efficiency, Long operating life, Noiseless operation, Higher speed ranges. 


\subsection{BLDC drives operation with inverter}

Basically it is an electronic motor and requires a three-phase inverter in the front end a shown in Fig.1. In self control mode the inverter acts like an electronic commutator that receives the switching logical pulse from the absolute position sensors. The drive is also known as an electronic commutated motor. Basically the inverter can operate in the following two modes.

- $2 \pi / 3$ angle switch-on mode

- Voltage and current control PWM mode

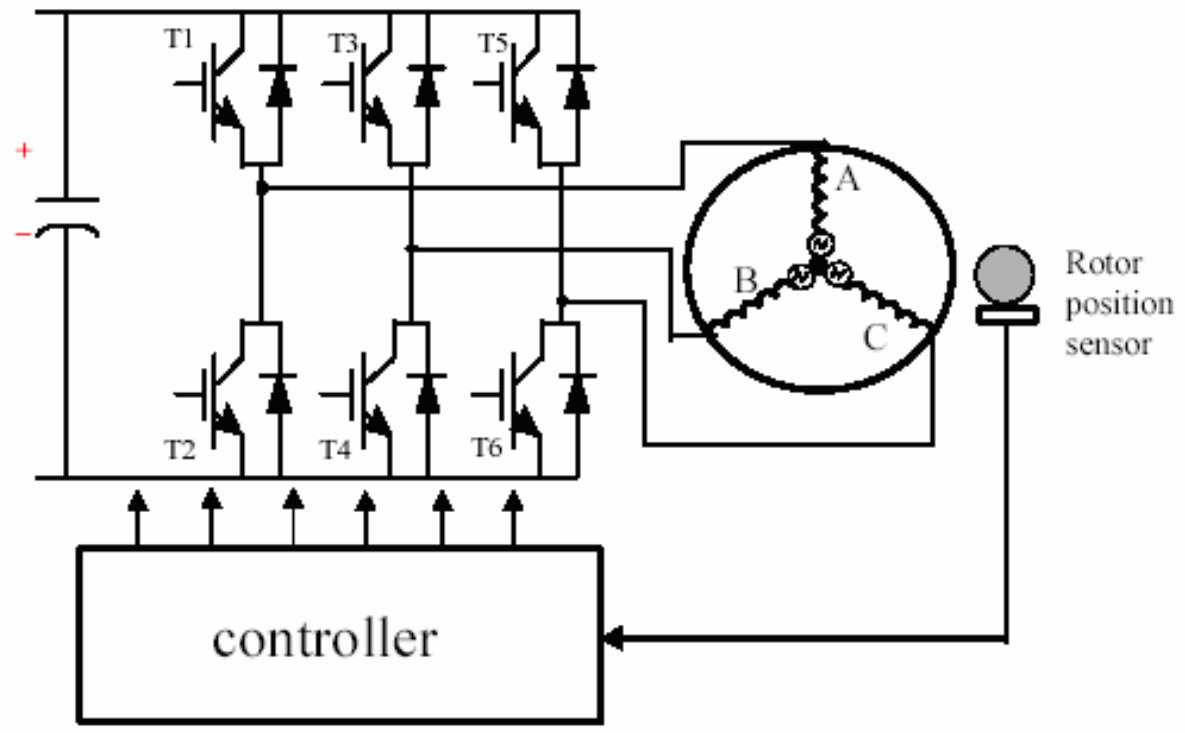

Fig.1. Brushless dc motor drive system

Table 1: Parameters of BLDC Motor

\begin{tabular}{|l|l|}
\hline TYPE & BLDC MOTOR \\
\hline VOLTAGE & 48 volts \\
\hline CURRENT & $15.625 \mathrm{~A}$ \\
\hline POWER & 750 watts \\
\hline SPEED & $1000 \mathrm{rpm}$ \\
\hline
\end{tabular}

\section{Implementation Of Flc In Vhdl}

Fuzzy logic control attempts to design the informal nature of the control design process. The Mamdani architecture is the way to design a fuzzy control system. The Mamdani architecture for fuzzy logic control is proposed by E. H. Mamdani in 1974, is that, in the absence of an explicit plant model and-or clear statement of control design objectives, informal knowledge of the operation of the given plant can be coded in terms of IFTHEN or condition-action, rules and form the basis for the linguistic control strategy, for example, a fuzzy rule IF Position Error is ok AND Angular Position is Middle THEN Control signal is No Action. where the Position Error and Angular Position are input variables and on the other hand, Control signal is an output variable. Ok, Middle and No Action are fuzzy sets, and the first two sets are input fuzzy sets and the last one is the output fuzzy set.

\subsection{Fuzzification}

The first component in the FLC is the fuzzifier that converts crisp inputs into a set of membership values in the interval $[-1,1]$ in the corresponding fuzzy sets. In this paper, Gaussian membership functions are used for two inputs Position Error and Angular Position. Each of inputs is represented by 3 membership functions, which are Negative, ok, Positive and Negative, Middle, Positive as shown in Fig.2 and Fig.3. 


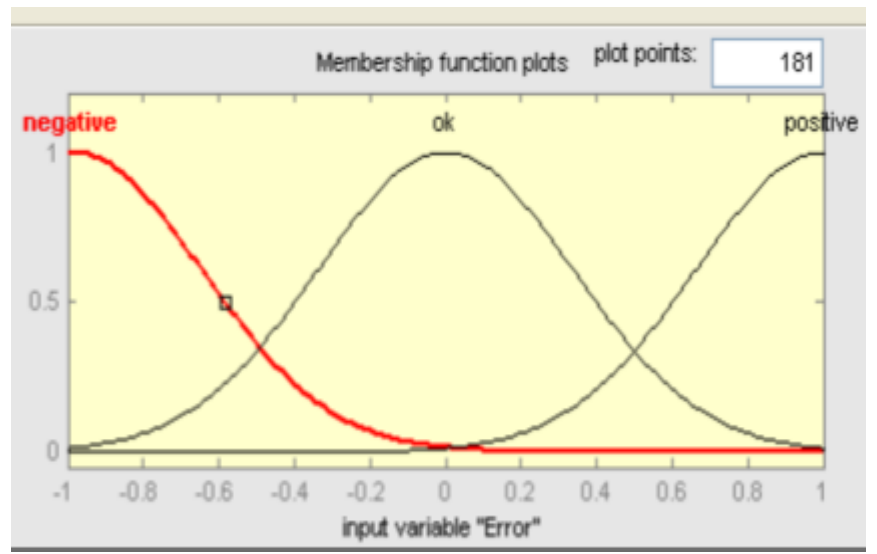

Fig.2. Membership Function for position error

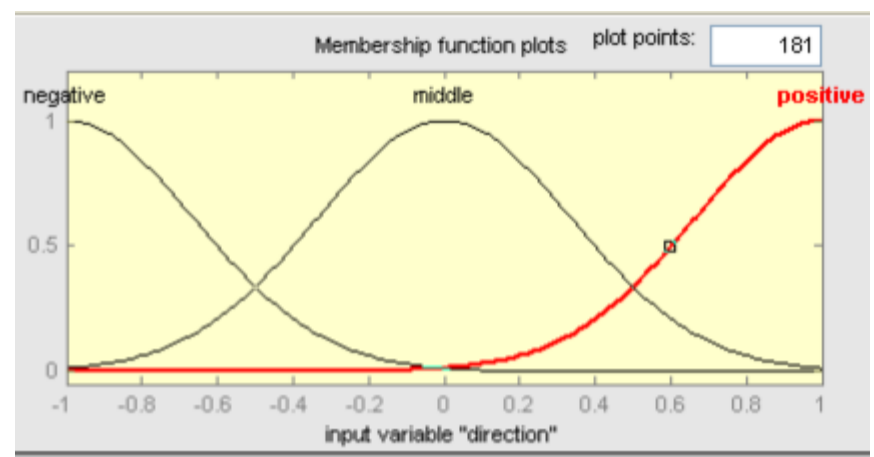

Fig.3. Membership Function for angular position

\subsection{Rule Inference}

The degree of membership is determined in the fuzzification stage. The next step is to create rules to decide what action should be taken in response to the given set of degree of membership function. The "AND" an "OR" fuzzy operators are best used for rules with multiple antecedents. The fuzzy operator, "OR" is used to evaluate the disjunction of the rules antecedents and "AND" is used to evaluate the conjunction of the rules antecedents. "AND" fuzzy operator is since it is required to evaluate the conjunction of the rules antecedents. Since "AND" is the minimum operation between multiple antecedents, the minimum function is used. The "OR" fuzzy operator also can be used when more than one rules involved with the same output. The rule base of system is defined in Table 1.

Table 1. Rule base of fuzzy-logic controller

\begin{tabular}{|l|l|l|}
\hline \multicolumn{2}{|c|}{ Input } & Output \\
\hline Position error & $\begin{array}{l}\text { Angular } \\
\text { position }\end{array}$ & Control signal \\
\hline Negative & Negative & Negative \\
\hline Ok & Middle & No action \\
\hline Positive & Positive & Positive \\
\hline
\end{tabular}

\subsection{Rule Evaluation}

Rule1: position $(1)<=$ maximum(minimum (u1(0), $\mathrm{u} 2(1))$,

minimum (u1(1), u2(0)));

A total number of rules that should be produced to describe the complete fuzzy control strategy can be calculated by multiplying the input membership function with the output membership function. Although there are number of possible rules, most of them can be discarded as long as the design is able to determine how the fuzzy control system should be operated.

\subsection{Defuzzification}

After the output for the each rule has been identified, the next step is to combine all the output into a single value that can used to control the motors. This process is done through defuzzification. The defuzzification technique used in Mamdani method is Centre of gravity. This is done by multiplying fuzzy output obtained from the rules evaluation with its corresponding singleton value, then sum of this value is 
divided by the sum of all fuzzy output obtained from the rules evaluation. The result from this calculation is the final single output which can be used to control the motor movements. Since there is no division symbol supported by Xilinx ISE Compiler, a divider circuit has to be designed to perform defuzzification.

\subsection{Simulation in MATLAB}

\section{Simulation And Results}

Simulations have been done for BLDC motor using FLC in MATLAB/SIMULINK. The simulink diagram of FLC is shown in Fig.4

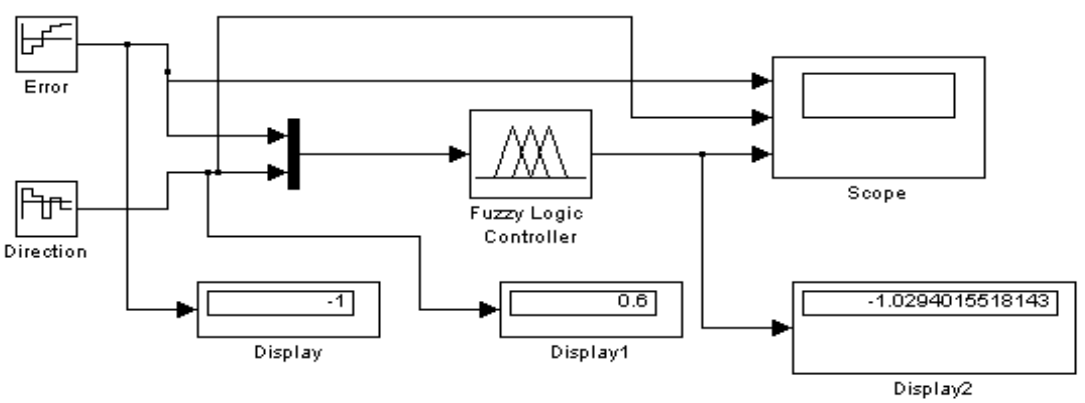

Fig.2. Simulink based block diagFig.5. Simulink Diagram of FLC ram of position control system

Fig.4. Simulink Diagram of FLC

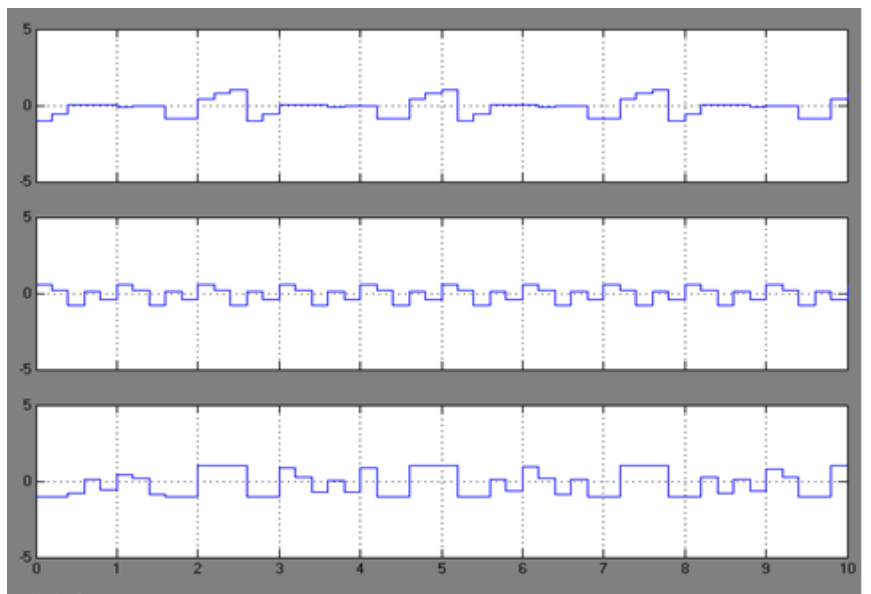

Fig.5. output waveform

\subsection{Simulation in Xilinx ISE}

Simulations have been done in Xilinx ISE.Fig.6 and Fig.7 shows that Test Bench Waveform and RTL view of FLC using VHDL respectively. In this figure the outputs have calculated according to input Position Error and Angular Position. 


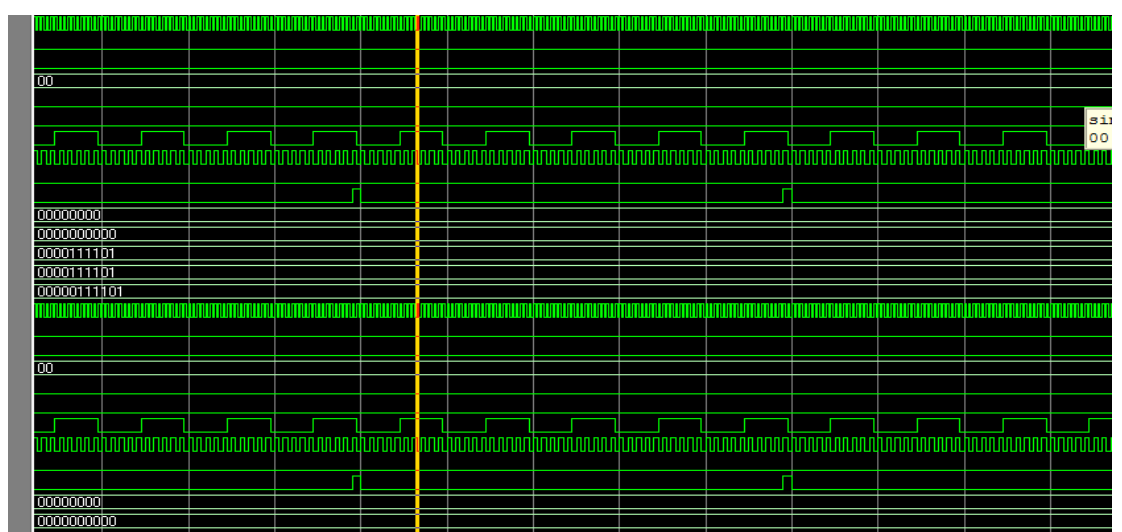

Fig.6. Waveforms of the implemented Fuzzy Logic with PWM Module

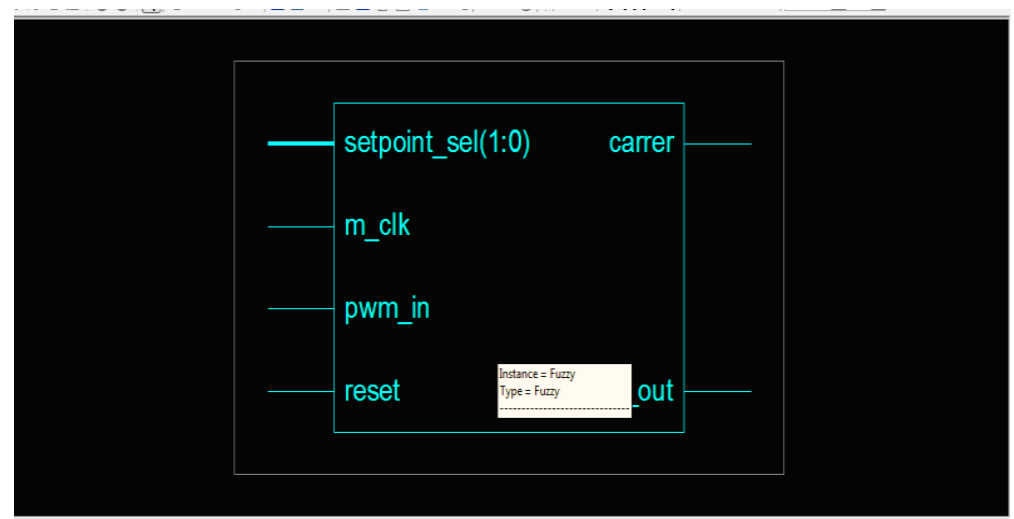

Fig.7. RTL view of FLC using VHDL

\section{Conclusion}

For designing FLC, a high- level modelling approach in VHDL have to be used. The advantages of this are reducing the design time, evaluation of the design functionality in a short time and quickly exploring of different design choices. Once the basic design of the fuzzy logic control system has been defined, the implementation of the fuzzy logic controller is very straight forward by coding each component of the fuzzy inference system in VHDL according to the design specifications. By simply changing some parameters in the codes and design constraint on the specific synthesis tool, one can experiment with different design circuitry to get the best result in order to satisfy the system requirement. From the results, we saw that the response of FLC using VHDL is better than the response of FLC using MATLAB. The peak overshoot and settling time both are better than others.

\section{References}

[1]. S. Assilian and E.H. Mamdani,-An Experiment in Linguistic Synthesis with Fuzzy Logic Controllerl, Int. Journal on Man machine studies. Vol. 7,pp. 1-13,1975.

[2]. R. Palm, - Scaling of Fuzzy Controller using the cross correlationll, IEEE Trans. Fuzzy Syst., Vol. 3, pp. 116-123, Feb. 1995

[3]. Sameep and Kuldip S. Rattan, - Implementation of a Fuzzy Controller on an FPGA using VHDLl, 22nd International Conference (NAFIPS), pp. 110-115, March 2003.

[4]. H. X. Li and H. B. Gatland, -Conventional Fuzzy Control and its enhancementll, IEEE Trans. Syst., Man, Cyber., Vol. 26, pp. 791$797,1996$.

[5]. Andrew Kusiak,"Fuzzy Logic", The University of Iowa, Iowa City 2004.

[6]. Yodyium Tipsuwan and Mo-Yuen Chow, -Fuzzy logic Microcontroller Implementation For DC Motor Speed controll, IEEE, pp. 1271-1276, 1999

[7]. Kwon, C.J., Han, W.Y., Kim, S.J. and Lee C.G., Speed controller with adaptive fuzzy tuning for BLDC motor drive under load variations. SICE Annual Conference, 3118-3121, 2003

[8]. Daijin Kim, - An Implementation of Fuzzy Logic Controller on the Reconfigurable FPGA System\|, IEEE Transaction on Industrial Electronics, Vol. 47, No. 3, pp. 703-715, June 2000.

[9]. Philip T. Voung, Asad M. Madni and Jim B. Vuong, - VHDL Implementation for a Fuzzy Logic Controller\|, World Automation Congress (WAC), July 24-26, 2006.

[10]. Clive Max field, Design Warrior Guide to FPGAs, Elsevier publications 2004.

[11]. Dr. Peter R. Wilson, Design recipes for FPGAs, Embedded technology series, Elsevier publication 2007.

[12]. Moe Shahdad,-An Overview of VHDL Language and Technologyll. 23rd Conference on Design Automation, pp.320-326, 1986. 